Herausgeberl Editors:
Im Auftrag der

Gesellschaft Deutscher Chemiker

B. Franck - K. Hafner · H. Musso $† \cdot$ H. Paulsen - E. Schmitz - U. Schöllkopf K. Schreiber $\cdot$ H. A. Staab
Redaktion/

Managing Editor:
R. Temme

mit W. Jung
Postfach 101161

D-6940 Weinheim

Tel. (06201) 602-255

\title{
JAHRESREGISTER/INDEX 1988
}

Verlag/Publisher:

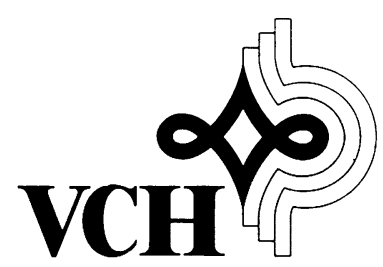

VCH Verlagsgesellschaft $\mathrm{mbH}$

Postfach 101161

D-6940 Weinheim

Federal Republic of Germany

Telefon (06201) 602-0

Telefax (06201) 602328

Telex 465516 vchwh d 


\title{
Neue chirale Hilfsgruppen: Gewinnung einer phenylazaanalogen Camphansäure nach veränderter $\mathrm{N} / \mathrm{O}$-Regioselektivität einer Ringverengungsreaktion
}

\author{
Klaus Th. Wanner \\ Institut für Pharmazie und Lebensmittelchemie der Universität München, \\ Sophienstraße 10, D-8000 München 2
}

Eingegangen am 26. Januar 1988

\section{New Chiral Auxiliary Groups: Formation of a Phenylaza Ana- logue of Camphanic Acid after Change of N/O-Regioselectivity of a Ring-Contraction Reaction}

By treating $\alpha$-bromo imide (-)-1 with sodium methylate an earlier observed mode of ring contraction is altered from $O$ - to $\mathrm{N}$-alkylation and the bicyclic lactam $(+)-3 \mathbf{b}$ is formed.

Im Rahmen unserer Untersuchungen über ,, asymmetrische elektrophile $\alpha$-Amidoalkylicrungen " $"$ haben wir uns für die Carbonsäure (+)-3c als chirale Hilfsgruppe interessiert. (+)-3c leitet sich von (-)-Camphansäure durch Austausch des Ringsauerstoffs durch eine Phenylaminogruppe ab. Man sollte erwarten, daß dieser hervorstehende Substituent dem Bicyclus (+)-3c ein hohes Induktionsvermögen verleiht. Wir versuchten, diese Verbindung aus dem $\alpha$-Bromimid (-)-1 durch Behandlung mit ethanolischer $\mathrm{KOH}$ in Dioxan darzustellen, haben aber das isomere Imidolacton (-)-5 als Hauptprodukt erhalten ${ }^{21}$.

Es gelang aber unter modifizierten Reaktionsbedingungen mit Tetrabutylammoniumhydroxid in Tetrahydrofuran, $(+)-3 \mathrm{c} z u$ isolieren, allerdings nur in geringen Mengen und nach einer umständlichen Aufarbeitung ${ }^{21}$. Für eine asymmetrische Synthese war jedoch cine ergiebigere Darstellung wünschenswert.
Bei einer genaueren Untersuchung der Umsetzung von (-)-1 mit ethanolischer $\mathrm{KOH}$ (in Dioxan) fanden wir nun in der Neutralfraktion vor allem den Ethylester (+)-3a der Lactamcarbonsäure $(+)-3 \mathbf{c}$, nicht aber den Ethylester von (-)-5. Somit ist festzustellen, daß unter den Carbonsäuren die Iminocamphansäure (-)-5 als Hauptprodukt zu finden ist, unter den Carbonsäurecstern dagegen das Phenylazaanaloge $(+)-\mathbf{3 a}$.

Das läßt sich folgendermaßen erklären: Beim Behandeln mit ethanolischer Kalilauge (in Dioxan) wird das $\alpha$-Bromimid (-)-1 durch Hydroxid zum Amid 4 gespalten (vermutlich über die Zwischenstufe 2, $\mathrm{R}=\mathrm{H}$ ); wird die Imidbindung von (-)-1 durch Ethanolat (aus der ethanolischen $\mathrm{KOH}$ ) gespalten, entsteht das AmidAnion 2 ( $\mathrm{R}=$ Ethyl). Das Amid 4 stabilisiert sich unter $O$-Alkylierung und Bildung von 5; das Amid-Anion $2(\mathrm{R}=$ Ethyl) setzt sich unter $N$-Alkylierung zum Lactam $(+)-3$ a um ${ }^{3)}$.

Auf Grund dieser Ergebnisse wurde (-)-1 mit Natriummethylat in Methanol umgesetzt, um so ausschließlich über das Amid-Anion $\mathbf{2}\left(\mathrm{R}=\mathrm{CH}_{3}\right)(+)-\mathbf{3 b}$ zu erhalten. Ein veränderter Reaktionsverlauf wegen vollständiger Protonierung durch das Lösungsmittel war dabei nicht zu erwarten ${ }^{4}$. Tatsächlich entstand dem DC zufolge ausschließlich der Methylester (+)-3b des gewünschten Lactams. (+)3b war identisch mit dem aus (+)-3c synthetisierten Methylester (+)-3b. Aus diesem ließ sich dann durch Erhitzen mit methanolischer $\mathrm{KOH}$ die Carbonsäure (+)-3c gewinnen.
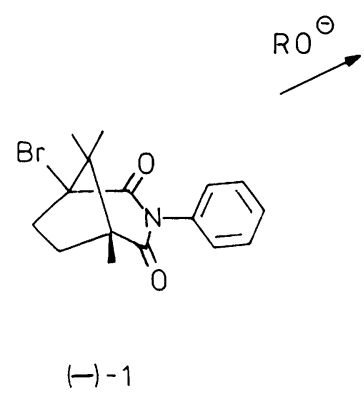$$
\text { . }
$$

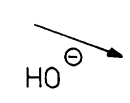

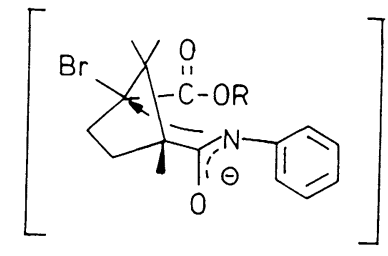

2

$$
\begin{aligned}
& 1 \\
& 1 \\
& 1
\end{aligned}
$$

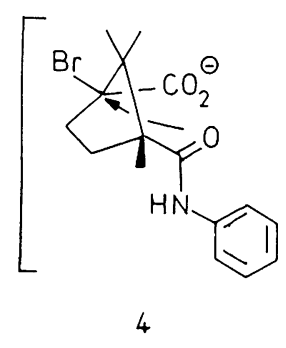

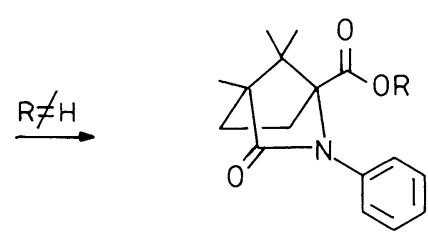
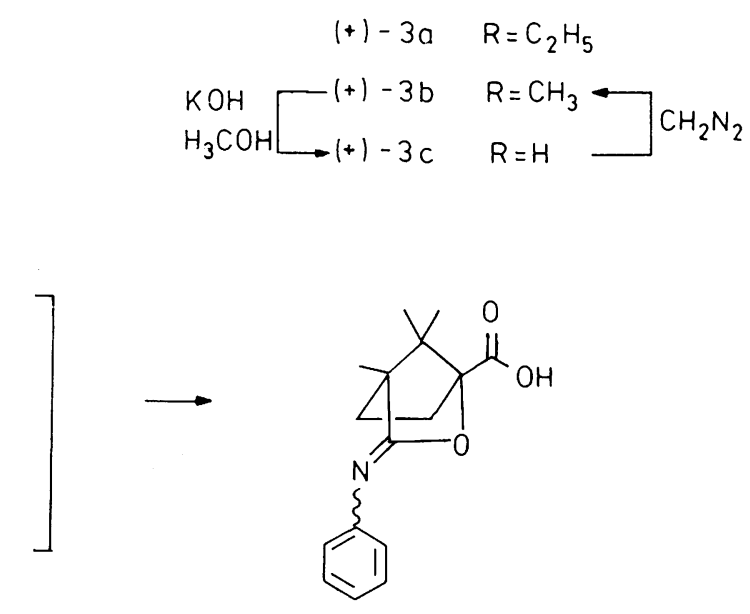

$(-)-5$ 
Mit dieser in ausreichenden Mengen herstellbaren Carbonsäure (+)-3c steht nun eine neue chirale Hilfsgruppe für asymmetrische Synthesen zur Verfügung.

Herrn Prof. F. Eiden danke ich für die großzügige Förderung und der Deutschen Forschungsgemeinschaft für die finanzielle Unterstützung dieser Arbeit. Dank schulde ich ferner Frau A. Kärtner und Frau $C$. Jung für Hilfe beim Experimentieren.

\section{Experimenteller Teil}

Schmp. (nicht korr): Schmelzpunktbestimmungsapparat nach Dr. Tottoli. - 'H-NMR-Spektren: A-60 (Varian) und WP 80 (Bruker); $\delta$-Skala (ppm), TMS int. Standard. $-{ }^{13}$ C-NMR-Spektren: WP 80 (Bruker); Lösungsmittel int. Standard. - IR-Spektren: Acculab 6 (Beckman); Flüssigkeiten als Film, Feststoffe als KBr-Preßlinge. Optische Drehungen: Lichtelektrisches Polarimeter Zeiss, 0.5-dmRohr. - CHN-Analysen: CHN-Rapid (Heraeus). - SC: „FlashChromatographie“"5).

Ethyl-〈(1S,4R)-4,7,7-trimethyl-3-oxo-2-phenyl-2-azabicyclo-

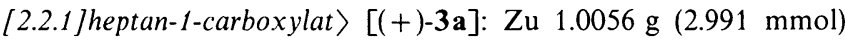
$(-)-1^{2)}$ in $200 \mathrm{ml}$ destilliertem Dioxan wurden innerhalb $40 \mathrm{~min}$ $12 \mathrm{ml} 0.5 \mathrm{M} \mathrm{KOH}$ (in EtOH) getropft. Nach $20 \mathrm{~h}$ wurde die trüb gewordene Lösung mit $1.5 \mathrm{ml} 2 \mathrm{~N} \mathrm{HCl}$ versetzt und i. Vak. eingeengt. Der Rückstand wurde in $50 \mathrm{ml}$ gesättigter $\mathrm{NaHCO}_{3}$-Lösung aufgenommen und mehrfach mit $\mathrm{Et}_{2} \mathrm{O}$ extrahiert. Die vereinigten $\mathrm{Et}_{2} \mathrm{O}$-Extrakte wurden getrocknet $\left(\mathrm{MgSO}_{4}\right)$ und i. Vak. eingeengt. Aus dem Rückstand wurde (+)-3a durch $\mathrm{SC}\left(\mathrm{CH}_{2} \mathrm{Cl}_{2} /\right.$ Ethylacetat $=97 / 3)$ abgetrennt $\left(\right.$ Zone mit kleinstem $\left.R_{\mathrm{f}}\right)$. Farblose Kristalle; Schmp. $55-56^{\circ} \mathrm{C},[\alpha]_{546}=+33,[\alpha]_{578}=+29(c=$ 0.93, $\left.\mathrm{CH}_{3} \mathrm{OH}\right)$, Ausb. $93.3 \mathrm{mg}(10 \%)$. - IR: $\tilde{v}=1720 \mathrm{~cm}^{-1}, 1495$, 1315. - 80-MHz- ${ }^{1} \mathrm{H}-\mathrm{NMR}\left(\mathrm{CDCl}_{3}\right): \delta=0.98\left(\mathrm{~s}, 3 \mathrm{H}, \mathrm{CH}_{3}\right), 1.00(\mathrm{t}$, $\left.J=7.3 \mathrm{~Hz}, 3 \mathrm{H}, \mathrm{CH}_{3} \mathrm{CH}_{2}\right), 1.06\left(\mathrm{~s}, 3 \mathrm{H}, \mathrm{CH}_{3}\right), 1.15\left(\mathrm{~s}, 3 \mathrm{H}, \mathrm{CH}_{3}\right)$, $1.53-2.43(\mathrm{~m}, 3 \mathrm{H}), 2.69(\mathrm{ddd}, J=5.5 / 10 / 13 \mathrm{~Hz}, 1 \mathrm{H}), 4.08$ (q, $J=$ $\left.7.3 \mathrm{~Hz}, 2 \mathrm{H}, \mathrm{OCH}_{2}\right), 7.01-7.34\left(\mathrm{~m}, 5 \mathrm{H}, \mathrm{C}_{6} \mathrm{H}_{5}\right) .-{ }^{13} \mathrm{C}-\mathrm{NMR}$ $\left(\mathrm{CDCl}_{3}\right): \delta=10.2,13.8,16.9,17.5\left(4 \mathrm{q}, 4 \mathrm{CH}_{3}\right), 29.8,30.8(2 \mathrm{t}$, $\mathrm{CH}_{2} \mathrm{CH}_{2}$ ), 54.4, 56.5 (2s, CMe, $\mathrm{CMe}_{2}$ ), $61.1\left(\mathrm{t}, \mathrm{OCH}_{2}\right), 121.8$ (d, C-2 Aromat), 124.7 (d, C-4 Aromat), 128.5 (d, C-3 Aromat), 137.9 (s, C-1 Aromat), 168.0 (s, COO), 177.1 (s, CON); $C-\mathrm{N}-\mathrm{C}=\mathrm{O}-$ Signal durch $\mathrm{CDCl}_{3}$ verdeckt.

$$
\begin{array}{ll}
\mathrm{C}_{18} \mathrm{H}_{23} \mathrm{NO}_{3} \text { (301.4) } & \text { Ber. C } 71.73 \mathrm{H} 7.69 \mathrm{~N} 4.65 \\
& \text { Gef. C } 71.76 \mathrm{H} 7.69 \mathrm{~N} 4.65 \\
& \text { Molmasse } 301 \text { (MS) }
\end{array}
$$

Methyl-〈(1S,4R)-4,7,7-trimethyl-3-oxo-2-phenyl-2-azabicyclo[2.2.1]heptan-1-carboxylat $\rangle[(+)-3 \mathrm{~b}]$ : a) $1.4765 \mathrm{~g}(4.391 \mathrm{mmol})$ $(-)-1^{2)}$, gelöst in $6.0 \mathrm{ml}$ absol. THF, wurden zusammen mit $3.8 \mathrm{ml}$ $1.5 \mathrm{M} \mathrm{NaOCH}_{3}$-Lösung in $\mathrm{CH}_{3} \mathrm{OH} 72 \mathrm{~h}$ bei Raumtemp. gerührt. Dann wurde das Lösungsmittel i. Vak. abgedampft, der Rückstand mit $\mathrm{H}_{2} \mathrm{O}(5.0 \mathrm{ml})$ und $\mathrm{Et}_{2} \mathrm{O}$ versetzt und die wäßrige Phase nach Sättigen mit $\mathrm{NaCl}$ noch mehrfach mit $\mathrm{Et}_{2} \mathrm{O}$ extrahiert. Die org. Extrakte wurden mit gesättigter $\mathrm{NaCl}$-Lösung gewaschen, getrocknet $\left(\mathrm{MgSO}_{4}\right)$ und i. Vak. vom Lösungsmittel befreit. Der Rückstand
$(1.030 \mathrm{~g}, 82 \%)$ wurde aus Diisopropylether umkristallisiert. Farblose Kristalle; Schmp. $76-77^{\circ} \mathrm{C},[\alpha]_{546}=+34,[\alpha]_{578}=+30(c=$ 1.29, $\left.\mathrm{CH}_{3} \mathrm{OH}\right)$, Ausb. $0.5755 \mathrm{~g}(46 \%) .-\mathrm{IR}: \tilde{v}=1740 \mathrm{~cm}^{-1}, 1710$, 1495, 1355. - 60-MHz- ${ }^{1} \mathrm{H}-\mathrm{NMR}\left(\mathrm{CDCl}_{3}\right): \delta=1.00\left(\mathrm{~s}, 3 \mathrm{H}, \mathrm{CH}_{3}\right)$, $1.08\left(\mathrm{~s}, 3 \mathrm{H}, \mathrm{CH}_{3}\right), 1.18\left(\mathrm{~s}, 3 \mathrm{H}, \mathrm{CH}_{3}\right), 1.57-3.00(\mathrm{~m}, 4 \mathrm{H}), 3.65(\mathrm{~s}, 3 \mathrm{H}$, $\left.\mathrm{OCH}_{3}\right), 7.00-7.60\left(\mathrm{~m}, 5 \mathrm{H}, \mathrm{C}_{6} \mathrm{H}_{5}\right) .-{ }^{13} \mathrm{C}-\mathrm{NMR}\left(\left[\mathrm{D}_{8}\right]\right.$ Dioxan): $\delta=$ 10.5, 17.0, $17.7\left(3 \mathrm{q}, 3 \mathrm{CH}_{3} \mathrm{C}\right), 30.1,31.3\left(2 \mathrm{t}, \mathrm{CH}_{2} \mathrm{CH}_{2}\right), 52.1$ (q, $\left.\mathrm{OCH}_{3}\right), 54.5,56.9\left(2 \mathrm{~s}, \mathrm{CMe}, \mathrm{CMe}_{2}\right), 77.5(\mathrm{~s}, \mathrm{C}-\mathrm{N}-\mathrm{C}=\mathrm{O}), 121.9$ (d, C-2 Aromat), 124.7 (d, C-4 Aromat), 129.0 (d, C-3 Aromat), 139.1 (s, C-1 Aromat), 169.2 (s, COO), 176.9 (s, CON).

$$
\begin{array}{ll}
\mathrm{C}_{17} \mathrm{H}_{21} \mathrm{NO}_{3} \text { (287.4) } & \text { Ber. C } 71.05 \mathrm{H} 7.37 \mathrm{~N} 4.87 \\
& \text { Gef. C } 71.23 \mathrm{H} 7.32 \mathrm{~N} 4.76 \\
& \text { Molmasse } 287 \text { (MS) }
\end{array}
$$

b) $\mathrm{Zu}$ einer Lösung von $26.3 \mathrm{mg}(0.0963 \mathrm{mmol})(+)-3 \mathrm{c}^{2)}$ in $10 \mathrm{ml}$ 90proz. $\mathrm{CH}_{3} \mathrm{OH}$ wurde bei Raumtemp. bis zur bestehenbleibenden Gelbfärbung etherische Diazomethanlösung getropft. Es wurde noch 30 min gerührt, dann i. Vak. eingeengt, mit $\mathrm{Et}_{2} \mathrm{O}$ versetzt, mit $0.1 \mathrm{~N} \mathrm{NaOH}(2 \times)$ und mit $\mathrm{H}_{2} \mathrm{O}(2 \times)$ gewaschen und getrocknet $\left(\mathrm{MgSO}_{4}\right)$. Nach Abdampfen des Lösungsmittels i. Vak. wurde zentrifugalchromatographisch $\left(\mathrm{CH}_{2} \mathrm{Cl}_{2} /\right.$ Ethylacetat $\left.=97 / 3\right)$ gereinigt. Farblose Kristalle; Ausb. $19.7 \mathrm{mg} \mathrm{(71 \% ).} \mathrm{Identität} \mathrm{mit} \mathrm{dem} \mathrm{unter}$ a) beschriebenen Produkt folgte aus ${ }^{1} \mathrm{H}-\mathrm{NMR}-,{ }^{13} \mathrm{C}-\mathrm{NMR}$ - und IRSpektren.

(1S,4R)-4,7,7-Trimethyl-3-oxo-2-phenyl-2-azabicyclo[2.2.1]heptan-1-carbonsäure $[(+)-3 \mathrm{c}]: 0.197 \mathrm{~g}(6.855 \mathrm{mmol})(+)-3 \mathbf{b}$ in $35 \mathrm{ml}$ $2 \mathrm{~N}$ methanolischer $\mathrm{KOH}$ wurden $3.5 \mathrm{~h}$ unter Rückfluß erhitzt. Anschließend wurde das Lösungsmittel i. Vak. abgedampft, der Rückstand in $\mathrm{H}_{2} \mathrm{O}$ gelöst und mit $\mathrm{Et}_{2} \mathrm{O}(2 \times)$ geschüttelt. Die Wasserphase wurde mit $2 \mathrm{~N} \mathrm{NCl}$ auf $\mathrm{pH} \approx 1$ eingestellt, worauf sich ein farbloser Niederschlag bildete, der auf Zusatz von $\mathrm{Et}_{2} \mathrm{O}$ in Lösung ging. Es wurde noch $3 \times$ mit $\mathrm{Et}_{2} \mathrm{O}$ extrahiert; die Extrakte (der sauren Wasserphase) wurden vereinigt, mit $\mathrm{H}_{2} \mathrm{O}$ gewaschen $(2 \times)$, getrocknet $\left(\mathrm{MgSO}_{4}\right)$ und i. Vak. zur Trockene eingeengt. Der Rückstand wurde aus Ethylacetat umkristallisiert. Farblose Kristalle; Ausb. $1.581 \mathrm{~g}(84 \%)$; Schmp. $242 \mathrm{C}$ (Lit. $\left.{ }^{2)} 241-251.5 \mathrm{C}\right) ;[\alpha]_{546}=$ $+57,[\alpha]_{578}=+51\left(c=0.37\right.$ in $\left.\mathrm{CH}_{3} \mathrm{OH}\right)\left\langle\right.$ Lit. $^{2)}[\alpha]_{546}=+54$, $[\alpha]_{578}=+49\left(c=0.205\right.$ in $\left.\left.\mathrm{CH}_{3} \mathrm{OH}\right)\right\rangle$.

\section{CAS-Registry-Nummern}

(-)-1: 113924-80-4 / (+)-3a: 113924-81-5 / (+)-3b: 113924-82-6/ $(+)-3 c: 113924-83-7$

1) K. Th. Wanner, A. Kärtner, Heterocycles 26 (1987) 921.

2) K. Th. Wanner, Arch. Pharm. (Weinheim, Ger.), im Druck.

3) Zur Regioselektivität bei der Alkylierung von Carbonsäureamiden und ihrer Anionen vgl.: B. C. Challis, J. A. Challis in Comprehensive Organic Chemistry (D. Barton, W. D. Ollis, Hrsg.), Bd. 2, S. 1011, Pergamon Press, Oxford 1979.

4) Vgl. hierzu die Säurestärke von Acetanilid und Methanol: J. Hine, M. Hine, J. Am. Chem. Soc. 74 (1952) 5266.

5) W. C. Still, M. Kahn, A. Mitra, J. Org. Chem. 43 (1978) 2923. 